\title{
Recurrent Bleedings in Newborn: A Factor VII Deficiency Case Report
}

\author{
Kim Cattivellia Cristina Distefano ${ }^{a}$ Lorenza Bonetti $^{b}$ Sophie Testa ${ }^{c}$ Simona Maria Siboni ${ }^{d}$ \\ Alessandro Plebania Carlo Poggiani ${ }^{\mathrm{e}}$ \\ a Pediatrics Clinic, University of Brescia, Spedali Civili di Brescia, Brescia, Italy; \\ bPediatrics Department, Istituti Ospedalieri di Cremona, Cremona, Italy; \\ ${ }^{\mathrm{c}}$ Hemostasis and Thrombosis Center, Istituti Ospitalieri di Cremona, Cremona, Italy; \\ ${ }^{\mathrm{d}}$ Angelo Bianchi Bonomi Hemophilia and Thrombosis Center, Fondazione IRCCS Ca' Granda Ospedale Maggiore Policlinico, Milan, Italy; \\ e Neonatal Intensive Care Unit, Istituti Ospedalieri di Cremona, Cremona, Italy
}

\section{Keywords}

Factor VII deficiency - Bleeding disorder - Newborn . Prophylaxis

\section{Summary}

Background: Major hemorrhages in newborns can be caused by several conditions, and knowledge of the differential diagnosis is essential in order to ensure prompt recognition and appropriate treatment. Case Report: We describe the case of a male newborn experiencing recurrent hemorrhages from the first days of life. Laboratory findings showed normal platelet count, hepatic function, and C-reactive protein. Coagulation tests detected an isolated prothrombin time (PT) prolongation and severe factor VII (FVII) deficiency. Conclusion: Inherited FVII deficiency is a rare autosomal recessive bleeding disorder. Clinical presentation is heterogeneous, and bleeding severity is not directly related to FVII levels. Acute bleeding episodes can be treated with human plasma-derived FVII (pdFVII) or recombinant activated FVII (rFVIIa). In case of severe deficiency, prophylaxis must be evaluated. Awareness of this condition is crucial in order to establish prompt diagnosis and treatment.

(c) 2018 S. Karger GmbH, Freiburg

\section{Introduction}

Major hemorrhages in newborns can be caused by several conditions, and knowledge of the differential diagnosis is essential in order to ensure prompt recognition and appropriate treatment.
Congenital diseases are less frequent than acquired coagulation disorders but should be considered. Inherited factor VII (FVII) deficiency is a rare autosomal recessive bleeding disorder caused by mutations in the FVII gene located on chromosome 13. Its prevalence is one on 300,000 people in Europe but can be higher in regions where consanguineous marriages are more common [1-3]. Clinical presentation can be variable, ranging from asymptomatic conditions to severe and potentially fatal bleedings.

\section{Case Report}

We report the case of a male newborn, born from consanguineous parents after physiological delivery. Family history was negative for bleeding disorders. During the second day of life, he presented epistaxis and hematemesis. Physical examination and vital signs were unremarkable. He was born in hospital and had received intramuscular vitamin K prophylaxis. No other drugs were administered, and maternal medications were excluded. Platelet count, hepatic function, and C-reactive protein (CRP) were normal. Routine coagulation tests detected an isolated increase of prothrombin time (PT) international normalized ratio (INR) (PT 5.93 INR; normal range 0,9-1.2 INR). Activated partial thromboplastin time (aPTT) and fibrinogen were normal. Coagulation factors were therefore checked, with the only evidence of severe FVII coagulation activity (FVII:C) deficiency (2\%; normal range $>50 \%$ ). Cardiac and abdominal ultrasounds were unremarkable. In the following days several other hemorrhagic episodes occurred. At first he experienced recurrent tongue bleedings and a spontaneous non-traumatic self-limiting paravertebral hematoma. Later, he presented melena, hematemesis, and macrohematuria. Melena and hematemesis were successfully treated with a therapeutic dose of recombinant activated FVII (rFVIIa) $(90 \mu \mathrm{g} / \mathrm{kg})$. After macrohematuria detection, a prophylactic treatment at a dosage of $30 \mu \mathrm{g} / \mathrm{kg} /$ twice a week was started [2].

Patient's parents were screened for coagulation disorders. His father has normal PT and mild FVII:C deficiency (38\%). His mother showed normal PT and normal FVII:C (103\%) 1 week after delivery. Two months later, FVII:C was $50 \%$, suggesting her heterozygous condition.

Genetic investigation revealed that our patient is homozygous for the known, pathogenic, splice site mutation IVS $2+1 \mathrm{G}>\mathrm{C}$. It consists in a guanine

\section{KARGER}

(C) 2018 S. Karger GmbH, Freiburg 
to cytosine substitution in nucleotide 3934 at position 1 of the IVS 2 donor splice site, probably causing the production of a truncated FVII deprived of an essential functional domain [4]. Both parents were confirmed to be heterozygous for the same mutation on chromosome 13

\section{Discussion}

We described a challenging case of recurrent neonatal hemorrhages. Coagulation tests are the first step in the diagnostic workup of pediatric bleeding (table 1). Acquired coagulation disorders are more frequent than congenital diseases. Among the acquired disorders, vitamin $\mathrm{K}$ deficiency, liver failure, and disseminated intravascular coagulation (DIC) are the main causes of early onset bleeding. Vitamin $\mathrm{K}$ deficiency is rarely seen today because prophylactic vitamin $\mathrm{K}$ is usually administered in the immediate newborn period, but it can occur when administration is delayed or in preterm infants [5]. Typically, PT and aPTT are both prolonged but aPTT can be normal in some cases. Coagulation factor test shows low levels of all vitamin K-dependent factors: factor II (FII), FVII, factor IX (FIX) and factor X (FX) [6]. The liver is responsible for the production of vitamin K-dependent factors, factor $\mathrm{V}(\mathrm{FV})$, fibrinogen, and anticoagulation factors (antithrombin III, protein C and S). Viral infections, hypoxia, shock, total parenteral nutrition, fetal hydrops, or metabolic disorders can lead to liver dysfunction and secondary early onset hemorrhages [7]. PT and aPTT are usually both prolonged, and the platelet count is typically low $[5,6]$. DIC is characterized by thrombocytopenia, prolonged PT and PTT, hypofibrinogenemia, and elevated fibrin degradation products (FDP) and D-dimer. The most frequent cause of DIC in newborns is sepsis $[5,6]$. Swallowed blood syndrome can also cause hematemesis and melena but usually it is not correlated to other bleedings and laboratory tests are normal [7].

Congenital bleeding disorders are rare, and the diagnosis can be difficult since newborns have lower levels of vitamin K-dependent factors and contact factors compared to adults [8]. Delivery-associated intracranial hemorrhages can occur in severe cases of hemophilia, although hemarthrosis and hematomas during the first year of life are the most typical presenting signs. In these cases, aPTT is usually prolonged while PT is normal and coagulation factors evaluation shows factor VIII (FVIII) deficiency in hemophilia A and FIX deficiency in hemophilia B [6]. Von Willebrand disease is the most common inherited bleeding disorder and is characterized by aPTT prolongation and mucosal hemorrhages. In type 3, von Willebrand factor (vWF) is completely absent, and phenotype is similar to severe hemophilia A [6]. Early onset can also occur in type 2, although the increased concentration of vWF and vWF multimers in newborns can make the diagnosis challenging [8]. Factor XI (FXI) deficiency is another rare bleeding disorder that determines aPTT prolongation [6,9]. Factor XIII (FXIII) deficiency usually presents with delayed umbilical cord hemorrhage. $\mathrm{PT}$ and aPTT are normal and diagnosis can be achieved by FXIII evaluation [6, 9]. a2-antiplasmin and plasminogen activator inhibitor-1 deficiency are extremely rare but should be considered when
Table 1. Laboratory findings in different causes of neonatal bleeding

\begin{tabular}{llll}
\hline & PT & aPTT & PLT \\
\hline Acquired bleeding disorders & & & \\
1. Vitamin K deficiency & $\uparrow$ & $\uparrow /=$ & $=$ \\
2. Liver failure & $\uparrow$ & $\uparrow$ & $\downarrow$ \\
3. DIC & $\uparrow$ & $\uparrow$ & $\downarrow$ \\
Inherited bleeding disorders & & & \\
1. Hemophilia A and B & $=$ & $\uparrow$ & $=$ \\
2. von Willebrand disease & $=$ & $\uparrow$ & $=/ \downarrow$ \\
3. Factor XI deficiency & $=$ & $\uparrow$ & $=$ \\
4. Factor XIII deficiency & $=$ & $=$ & $=$ \\
5. a2-antiplasmin deficiency & $=$ & $=$ & $=$ \\
6. Plasminogen activator inhibitor 1 & $=$ & $=$ & $=$ \\
$\quad$ deficiency & & & $=$ \\
7. Afibrinogenemia & $\uparrow$ & $\uparrow$ & $=$ \\
8. Factor II deficiency & $\uparrow$ & $\uparrow$ & $=$ \\
9. Factor V deficiency & $\uparrow$ & $\uparrow$ & $=$ \\
10. Factor X deficiency & $\uparrow$ & $\uparrow$ & $=$ \\
11. Factor VII deficiency & $\uparrow$ & $=$ & $=$ \\
\hline
\end{tabular}

PT = Prothrombin time; aPTT =activated partial thromboplastin time; PLT = platelets; DIC = disseminated intravascular coagulation.

PT and aPTT are normal [6]. PT and aPTT are instead both prolonged in afibrinogenemia and in FII, FV and FX deficiency [5, 6, 9]. In our patient, the combination of isolated PT prolongation, normal liver function and platelet count, and absence of an active inflammatory process led to the suspect and diagnosis of congenital FVII deficiency.

FVII deficiency is an uncommon heritable bleeding disorder with heterogeneous clinical presentation. Patients can be asymptomatic or experience different types of bleeding, with the most frequent being epistaxis, gum bleedings, menorrhagia, ecchymoses, and hematomas [3]. Hemarthrosis and life-threatening cerebral and gastrointestinal hemorrhages can also occur [3]. Because of the poor correlation between FVII:C levels and bleeding phenotype [10], the classification of FVII deficiency is usually based on clinical features. Age of onset is a crucial element, being a significant predictor of severe disease $[1,2]$. Considering the occurrence of early onset, multifocal major bleedings and the extremely low FVII:C, our patient was classified as a severe case.

Altered FVII plasma levels can be caused by 250 different mutations, and the majority of them are missense mutations [11]. Homozygous and compound heterozygous patients experience the most severe forms of the disease and carry a significant risk of premature death [11]. Homozygosity for the IVS $2+1 \mathrm{G}>\mathrm{C}$ mutation, which was detected in our patient, has been previously described only in one case, with fatal outcome at the age of 3 months because of intracranial hemorrhage $[4,12]$.

Specific treatment is indicated in the acute phase of clinically significant major bleedings. Prophylactic treatment is usually recommended for patients affected by severe phenotype, in children aged less than 2 years with FVII:C below 1\%, and in cases of recurrent hemarthrosis [2]. 
Several treatment options are available, both for therapeutic and prophylactic aim. Fresh frozen-plasma (FFP) is a widely available and low-cost therapeutic option, although FVII concentrations are low. The main drawbacks of FFP are possible circulatory overload due to high volume infusion and risk of viral infection transmission. Plasma complex concentrates represent an alternative but, because of the high content of several other vitamin K-dependent factors, these compounds increase the risk of arterial or, more often, venous thrombosis.

Plasma-derived FVII (pdFVII) and rFVIIa are equally effective for acute bleeding and prophylactic purpose $[1,2]$. Choice is driven by product availability and careful evaluation of risk/benefit ratio based on patient's history. Main drawbacks of pdFVII are risk of pathogen transmission, although limited by inactivation procedures, and need for large volume infusion that may lead to circulatory overload especially in newborns. Thrombosis and inhibitor development are the main side effects of rFVII [14]. rFVII should therefore be avoided in subjects with thrombosis risk factors, such as surgery and advanced age $[13,14]$. The prevalence of rFVII inhibitors was estimated to be around $1.8 \%$ [14]. This low rate could be explained by the rarity of severe gene defects such as large deletions $[14,15]$. Anaphylactic reactions have never been reported [14], and presence of inhibitors does not influence the efficacy of prophylaxis $[14,15]$. rFVII has a shorter half-life compared to pdFVII but the larger volume of distribution may explain its long lasting effect [16]. Considering the young age of our patient and the absence of thrombosis risk factors, rFVIIa was chosen $[1,2]$.
Prophylaxis with rFVIIa in a schedule based on $20-30 \mu \mathrm{g} / \mathrm{kg}$ of rFVIIa twice or three times a week has been described as the therapeutic regimen with the best outcomes in terms of reduction of bleeding's severity and frequency [2]. Our patient underwent early prophylaxis with a $30 \mu \mathrm{g} / \mathrm{kg}$ of rFVIIa twice a week schedule. A Baby Port-A-Cath was inserted in order to guarantee a persistent intravenous access. So far the prophylaxis has been successful to prevent further major bleedings in the following 2 years, with no side effects.

\section{Conclusion}

Multifocal spontaneous bleedings in the first days of life associated with prolonged PT should raise the suspicion of a FVII deficiency. Severe phenotypes are characterized by early onset, major bleedings, and low FVII:C values, although laboratory tests not always correlate with clinical presentation. To our knowledge, this is the first report of FVII deficiency due to homozygous state for IVS2 $+1 \mathrm{G}>\mathrm{C}$ mutation surviving longer than 3 months.

Prophylactic rFVIIa or pdFVII administration is mandatory in severe cases to avoid life-threatening bleedings. Awareness of this uncommon but potentially treatable condition is crucial, in order to establish prompt diagnosis and treatment.

\section{Disclosure Statement}

All authors declare to have no competing interests. No funding applicable for this case report.

\section{References}

1 Mariani G, Napolitano M, Dolce A, Pérez Garrido R, Batorova A, Karimi M, Platokouki H, Auerswald G, Bertrand AM, Di Minno G, Schved JF, Bjerre J, Ingerslev J, Sørensen B, Ruiz-Saez A; Seven Treatment Evaluation Registry; International Factor VII Deficiency Study Group: Replacement therapy for bleeding episodes in factor VII deficiency. a prospective evaluation. Thromb Haemost 2013;109:238-247.

2 Napolitano M, Giansily-Blaizot M, Dolce A, Schved JF, Auerswald G, Ingerslev J, Bjerre J, Altisent C, Charoenkwan P, Michaels L, Chuansumrit A, Di Minno G, Caliskan U, Mariani G: Prophylaxis in congenital factor VII deficiency: indications, efficacy and safety. Results from the Seven Treatment Evaluation Registry (STER). Haematologica 2013;98:538-544.

3 Di Minno MN, Dolce A, Mariani G; STER Study Group: Bleeding symptoms at disease presentation and prediction of ensuing bleeding in inherited FVII deficiency. Thromb Haemost 2013;109:1051-1059.

4 Wulff K, Herrmann FH: Twenty two novel mutations of the factor VII gene in factor VII deficiency. Hum Mutat 2000;15:489-496.

5 Jaffray J, Young G, Ko RH: The bleeding newborn: a review of presentation, diagnosis, and management. Semin Fetal Neonatal Med 2016;21:44-49.

6 Khair K, Liesner R: Bruising and bleeding in infants and children - a practical approach. Br J Haematol 2006;133:221-231.
7 Orkin SH, Nathan DG, Ginsburg D, Look AT, Fisher DE, Lux S IV (eds): Nathan and Oski's Hematology and Oncology of Infancy and Childhood, 8th ed. Philadelphia, Elsevier Saunders, 2014.

8 Kenet G, Chan AK, Soucie JM, Kulkarni R. Bleeding disorders in neonates. Haemophilia. 2010;16(suppl 5): 168-175.

9 Palla R, Peyvandi F, Shapiro AD: Rare bleeding disorders: diagnosis and treatment. Blood 2015;125:20522061.

10 Peyvandi F, Palla R, Menegatti M, Siboni SM, Halimeh S, Faeser B, Pergantou H, Platokouki H, Giangrande P, Peerlinck K, Celkan T, Ozdemir N, Bidlingmaier C, Ingerslev J, Giansily-Blaizot M, Schved JF, Gilmore R, Gadisseur A, Benedik Dolnicar M, Kitanovski L, Mikovic D, Musallam KM, Rosendaal FR, on behalf of the European Network of Rare Bleeding Disorders (EN-RBD) group: Coagulation factor activity and clinical bleeding severity in rare bleeding disorders: results from the European Network of Rare Bleeding Disorders. J Thromb Haemost 2012;10:615-621.

11 Herrmann FH, Wulff K, Auerswald G, Schulman S, Astermark J, Batorova A, Kreuz W, Pollmann H, RuizSaez A, De Bosch N, Salazar-Sanchez L; Greifswald Factor FVII Deficiency Study Group: Factor VII deficiency: clinical manifestation of 717 subjects from Europe and Latin America with mutations in the factor 7 gene. Haemophilia 2009;15:267-280.
12 Tamary H, Fromovich-Amit Y, Shalmon L, Zaizov R, Yaniv I, Klar A, Peretz H, Brenner B, Lanir N, Zivelin A, Seligsohn U: Molecular characterization of four novel mutations causing factor VII deficiency. Hematol J 2000;1:382-389.

13 Girolami A, De Marinis GB, Bonamigo E, Lombardi AM: Recombinant FVIIa concentrate-associated thrombotic events in congenital bleeding disorders other than hemophilias. Hematology 2012;17:346-349.

14 Napolitano M, Dolce A, Batorova A: Replacement therapy in inherited factor VII deficiency: occurrence of adverse events and relation with surgery. Haemophilia 2015;21:e513-e517.

15 Ingerslev J, Christiansen K, Sørensen B: International Registry on Factor VII Deficiency (IRF7) Steering Committee. Inhibitor to factor VII in severe factor VII deficiency: detection and course of the inhibitory response. J Thromb Haemost 2005;3:799-800.

16 Mathijssen NCJ, Masereeuw R, Holme PA, van Kraaij MG, Laros-van Gorkom BA, Peyvandi F, van Heerde WL: Increased volume of distribution for recombinant activated factor VII and longer plasma-derived factor VII half-life may explain their long lasting prophylactic effect. Thromb Res 2013;132:256-262. 